\title{
Femtosecond Spin Current Pulses Generated by the Nonthermal Spin-Dependent Seebeck Effect and Interacting with Ferromagnets in Spin Valves
}

\author{
Alexandr Alekhin, ${ }^{1, \dagger}$ Ilya Razdolski, ${ }^{1}$ Nikita Ilin, ${ }^{1, \$}$ Jan P. Meyburg, ${ }^{2}$ Detlef Diesing, ${ }^{2}$ Vladimir Roddatis, ${ }^{3}$ Ivan Rungger, ${ }^{4, \S}$ \\ Maria Stamenova, ${ }^{4}$ Stefano Sanvito, ${ }^{4}$ Uwe Bovensiepen, ${ }^{5}$ and Alexey Melnikov ${ }^{1,6, *}$ \\ ${ }^{1}$ Department of Physical Chemistry, Fritz Haber Institute of the Max Planck Society, Faradayweg 4-6, 14195 Berlin, Germany \\ ${ }^{2}$ Faculty of Chemistry, University of Duisburg-Essen, Universitätsstr. 5, 45117 Essen, Germany \\ ${ }^{3}$ Institute of Materials Physics, University of Goettingen, Friedrich-Hund-Platz 1, 37077 Goettingen, Germany \\ ${ }^{4}$ School of Physics and CRANN, Trinity College Dublin, Dublin 2, Ireland \\ ${ }^{5}$ Faculty of Physics, University of Duisburg-Essen, Lotharstr. 1, 47057 Duisburg, Germany \\ ${ }^{6}$ Institute of Physics, Martin Luther University Halle-Wittenberg, Von-Danckelmann-Platz 3, 06120 Halle, Germany
}

(Received 25 July 2016; published 6 July 2017)

\begin{abstract}
Using the sensitivity of optical second harmonic generation to currents, we demonstrate the generation of 250-fs long spin current pulses in $\mathrm{Fe} / \mathrm{Au} / \mathrm{Fe} / \mathrm{MgO}(001)$ spin valves. The temporal profile of these pulses indicates ballistic transport of hot electrons across a sub-100 nm Au layer. The pulse duration is primarily determined by the thermalization time of laser-excited hot carriers in Fe. Considering the calculated spin-dependent $\mathrm{Fe} / \mathrm{Au}$ interface transmittance we conclude that a nonthermal spin-dependent Seebeck effect is responsible for the generation of ultrashort spin current pulses. The demonstrated rotation of spin polarization of hot electrons upon interaction with noncollinear magnetization at $\mathrm{Au} / \mathrm{Fe}$ interfaces holds high potential for future spintronic devices.
\end{abstract}

DOI: 10.1103/PhysRevLett.119.017202

Optimization and control of spin currents (SC) and their interaction with magnetic constituents in heterostructures on a femtosecond time scale is key for future terahertz spintronics applications. Although electronic transport through a ferromagnet (FM), as described by Mott's two current model [1], generates a spin-polarized current, its density is intrinsically limited by Joule losses. The discovery of the spin-dependent Seebeck effect (SdSE), where thermal gradients over a bulk FM [2] or across an interface to a normal metal [3] generate SCs, opened a path towards overcoming such limitations. Indeed, shortlived thermal gradients can produce short $(\sim 100 \mathrm{ps}) \mathrm{SC}$ pulses at densities exceeding the static Joule limit, as recently demonstrated upon laser excitation of spin-valve structures [4].

Creating highly energetic electrons [5-9], femtosecond laser excitation is promising for SC pulse generation on subpicosecond time scales, before the electron-electron [9] and electron-lattice [8] equilibration is reached. Superdiffusive transport of laser-excited spin-polarized hot carriers on a femtosecond time scale $[10,11]$ was evidenced in a plethora of experiments [12-18]. However, unraveling the underlying microscopic picture requires understanding the influence of the electron dynamics in a FM, the scattering in metallic layers, and the properties of interfaces on the SC pulse. For this, a direct SC detection is highly desirable yet challenging in the presence of laser-induced magnetization dynamics [19].

In this Letter we argue that under nonequilibrium conditions, the SdSE at interfaces can lead to the efficient generation of femtosecond SC pulses paving the way for future $\mathrm{THz}$ spintronics. Employing a nonlinear-optical approach, we demonstrate the generation of these pulses at the $\mathrm{Fe} / \mathrm{Au}$ interface and Stern-Gerlach-like spatial spin separation upon their interaction with an orthogonal FM magnetization. The latter facilitates the development of nondissipative metallic spin polarizers and rotators for ultrafast spintronics.

In SdSE, spin transport is determined by gradients of the electron distribution function $\nabla f(\mathcal{E})$, which are drastically enhanced at interfaces. The difference $\Delta f(\mathcal{E})$ across the interface enables the flux of electrons $(e)$ at energy $\mathcal{E}>\mathcal{E}_{F}$ or holes $(h)$ at $\mathcal{E}<\mathcal{E}_{F}$, where $\mathcal{E}_{F}$ is the Fermi energy. A charge current $\mathbf{j}$ emerges when the total fluxes of $e$ and $h$ are unequal due to an asymmetry (with respect to $\mathcal{E}_{F}$ ) of $\Delta f(\mathcal{E})$, conductance [20], or the interface transmittance $T$, known as the Seebeck effect. If $\mathbf{j}_{\uparrow} \neq \mathbf{j}_{\downarrow}$ in the majority and minority subbands of FM, the resulting $\mathbf{j}=\mathbf{j}_{\uparrow}+\mathbf{j}_{\downarrow}$ is accompanied by the SC $\mathbf{j}^{\mathrm{S}} \propto \mathbf{j}_{\uparrow}-\mathbf{j}_{\downarrow}$.

Now we analyze $\mathrm{SdSE}$ at the $\mathrm{Fe} / \mathrm{Au}(001)$ interface for thermal and nonthermal $f(\mathcal{E})$. From $a b$ initio quantum transport calculations [21] we have obtained the momentum-averaged interface transmittance $T_{F A}$ for spin- $\uparrow$ and $-\downarrow$ carriers moving from $\mathrm{Fe}$ to $\mathrm{Au}$ [Fig. 1(a)]. In the thermal case, the carriers at energies within $k_{B} T_{e} \lesssim$ $100 \mathrm{meV}$ around $\mathcal{E}_{F}$ are responsible for the transport $[4,20]$, where $T_{e}(z)$ is the electron temperature. Therefore, the thermal SdSE originates from the slope of transmittance $\nabla_{\mathcal{E}} T_{F A}\left(\mathcal{E}_{F}\right)$ [Fig. 1(a)] and $\Delta f(\mathcal{E})$ determined by the temperature gradient $\nabla_{z} T_{e}$ across the interface. 

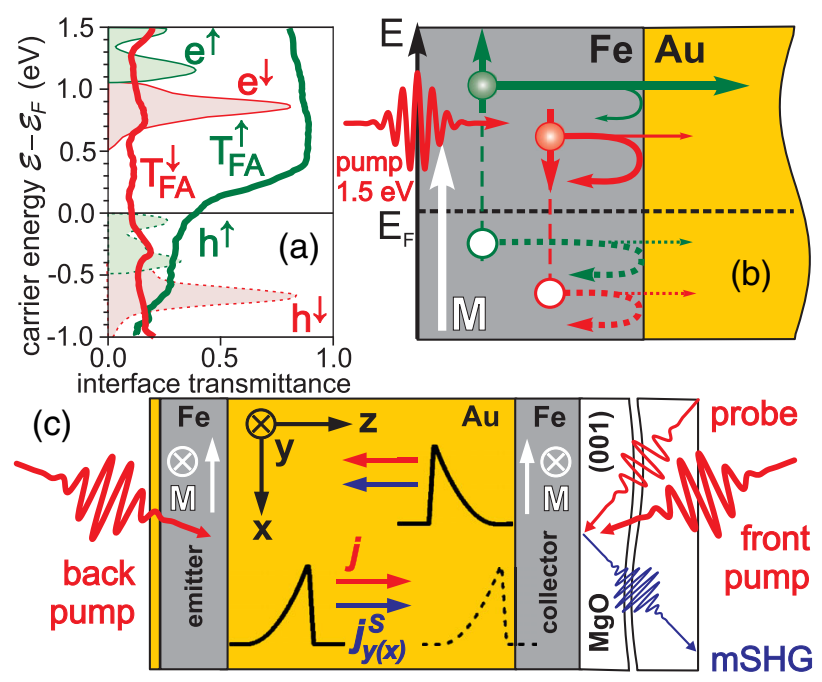

FIG. 1. (a) Calculated momentum-averaged transmittance of the $\mathrm{Fe} / \mathrm{Au}$ interface for majority $\left(T_{F A}^{\uparrow}\right)$ and minority $\left(T_{F A}^{\downarrow}\right)$ carriers moving from $\mathrm{Fe}$ to $\mathrm{Au}$. Shaded areas reproduce schematically the spectrum of primary carriers excited by the $1.5 \mathrm{eV}$ pump [14]. (b) Nonthermal spin-dependent Seebeck effect: among the hot carriers excited in Fe, only majority electrons with the energy above $0.3 \mathrm{eV}$ are effectively transmitted into $\mathrm{Au}$. (c) Experimental scheme: switching between the front and back pump changes the direction of currents in the Au spacer while the magnetization $\mathbf{M}$ in the pumped Fe layer set along one of the two equivalent easy axes $\hat{\mathbf{x}}=[100]$ and $\hat{\mathbf{y}}=[010]$ defines the spin polarization.

A much broader nonthermal $f(\mathcal{E})$ is formed by $1.5 \mathrm{eV}$ laser excitation of Fe [shaded areas in Fig. 1(a)] [14]. High optical reflectance ensures the absence of excited carriers in Au providing much larger $\Delta f$ than in the thermal case. Excited into the $s p$ band of Fe [14,24], $e^{\uparrow}$ have much larger transmittance [Figs. 1(a),1(b)] than other carriers resided in the $d$ band which poorly matches the $s p$ band of Au. Moreover, much higher velocity of $e^{\uparrow}$ in Fe [25] provides their better transport towards the interface. Thus, an efficient injection of $e^{\uparrow}$ into Au [Figs. 1(b),1(c)] results in SC pulses with the spin component of $j_{z, i}^{S}$ along $\hat{\mathbf{i}}=\hat{\mathbf{x}}, \hat{\mathbf{y}}$ set by the Fe magnetization and the average electron velocity $\mathbf{v}$ along $\hat{\mathbf{z}}$ [Fig. 1(c)]. Noteworthy, a similar spin-dependent interface transmittance can be expected in any itinerant FM/noble metal bilayer [26] facilitating demagnetization of the FM by superdiffusive spin transport $[10,11]$.

In this nonthermal SdSE, the collective displacement of "cold" non-spin-polarized electrons near $\mathcal{E}_{F}$ in Au partially screens the charge but not the spin component of the $e^{\uparrow}$ transport. Therefore, compared to the thermal case, SC pulses of much larger amplitude can be expected while the net charge transfer is suppressed. Furthermore, the pulse duration here will be primarily determined by the electron thermalization time in FM $\tau_{\text {th }}^{\mathrm{FM}} \lesssim 0.5$ ps [9,27]: once the nonthermal $f(\mathcal{E})$ [Fig. 1(a)] is relaxed to the Fermi-Dirac distribution, the remaining SC is of purely thermal origin.

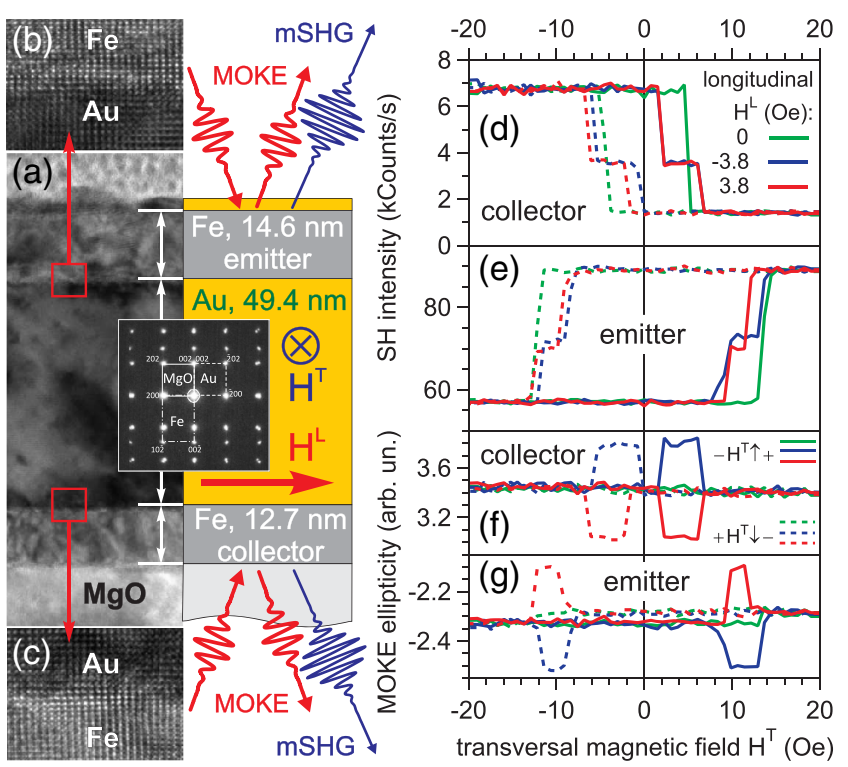

FIG. 2. (a)-(c) Cross-section transmission electron microscopy images of $\mathrm{Fe} / \mathrm{Au} / \mathrm{Fe} / \mathrm{MgO}(001)$ stack capped with $3 \mathrm{~nm}$ of $\mathrm{Au}$ and the experimental scheme. Electron diffraction (inset) reveals epitaxial growth of layers. Hysteresis loops measured by $\mathrm{mSHG}$ (d),(e) in $p$-in, $p$-out polarization geometry and by $p$-in MOKE (f), (g) in the bottom (collector) (d),(f) and top (emitter) (e), (g) Fe layers vs the transversal external magnetic field $H^{T} \| \hat{\mathbf{y}}$ for the indicated values of the longitudinal field $H^{L} \| \hat{\mathbf{x}}$, as shown in (a).

The thus expected generation of ultrashort and intense SC pulses is demonstrated below.

To achieve steady control of propagation direction and spin polarization of SC pulses, we combine the spin-valve concept with the pump-probe approach developed in Ref. [14]; see Fig. 1(c). The two Fe layers in the epitaxial $\mathrm{Fe} / \mathrm{Au} / \mathrm{Fe} / \mathrm{MgO}(001)$ stack [Fig. 2(a)] are magnetically decoupled and can be optically excited and probed independently owing to the $50 \mathrm{~nm}$-thick Au spacer. The samples were fabricated following Ref. [14] and examined with transmission electron microscopy, see Figs. 2(a)-2(c). The excellent epitaxial quality and flatness of interfaces, are essential for comparison with the $a b$ initio calculations performed for an atomically sharp interface.

Magnetoinduced second harmonic generation (mSHG) $[28,29]$ is a promising tool for direct SC detection owing to its high temporal resolution and sensitivity to the currentdriven symmetry breaking, as demonstrated in GaAs [30,31] and multilayer graphene [32]. We performed alloptical experiments in either the front or back pump-probe scheme shown in Fig. 1(c). The $800 \mathrm{~nm}, 14 \mathrm{fs}, 1 \mathrm{MHz}$ output of a cavity-dumped Ti:sapphire oscillator (Mantis, Coherent) was split at a power ratio 4:1 into pump and probe pulses both focused into an $\sim 10 \mu \mathrm{m}$ spot resulting in a pump fluence of $\sim 10 \mathrm{~mJ} / \mathrm{cm}^{2}$. The magneto-optical Kerr effect (MOKE) was measured with a balanced twophotodiode detector while the second harmonic signal was registered by a photomultiplier [14]. 


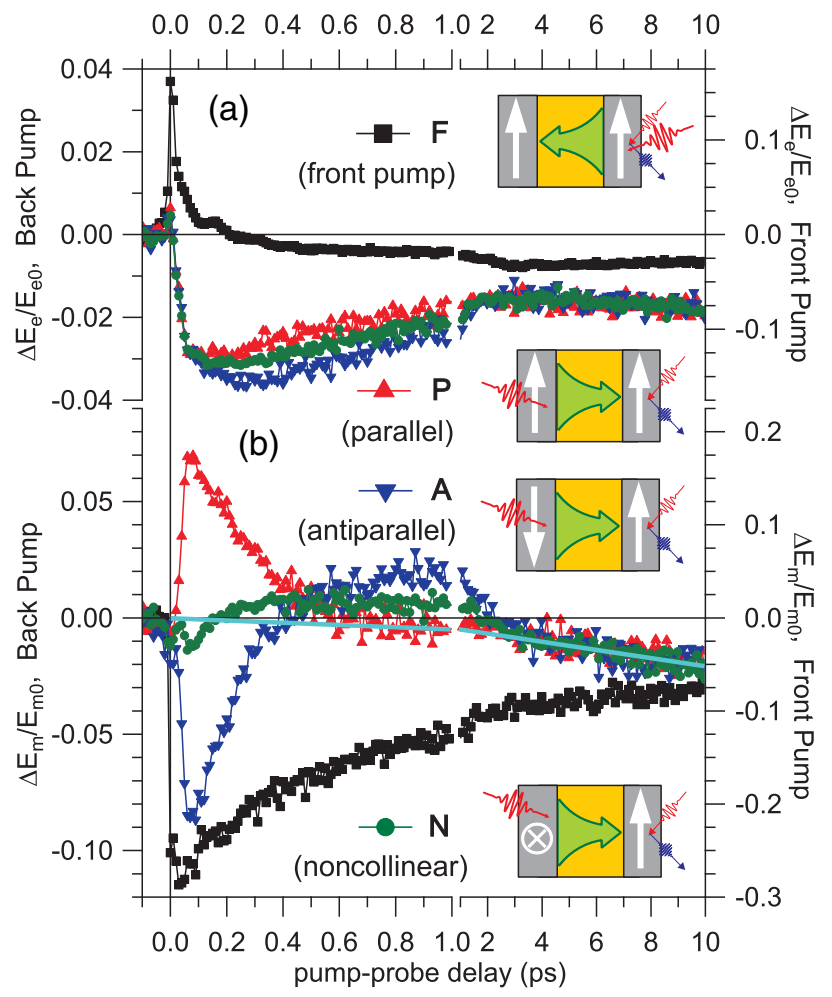

FIG. 3. Variations of nonmagnetic and current-induced (a) and magnetization- and SC-induced (b) components of the second harmonic electric field observed in $p$-in, $p$-out polarization geometry. SC vs $\mathbf{M}$ configurations for the front $(F)$ and back $(P, A, N)$ pumping are shown in the legend [cf. Fig. 1(c)]. The solid line represents the common background due to heatinginduced demagnetization.

The second harmonic field $\mathbf{E}_{\mathbf{2} \omega}=\mathbf{E}_{\mathbf{e}}+\mathbf{E}_{\mathbf{m}}$ consists of "electronic" $\mathbf{E}_{\mathbf{e}}$ and magnetoinduced $\mathbf{E}_{\mathbf{m}}$ terms which are, respectively, independent of and proportional to the magnetization $\mathbf{M}$ [28]. In this work $(x, z)$ is the plane of incidence and we use $p$-in, $p$-out polarization geometry. Then, $\mathbf{E}_{\mathbf{m}}$ is proportional to $M_{y}$, the transversal magnetization component $[14,28]$ while the MOKE is sensitive to the longitudinal $M_{x}$ [33]. In order to set the magnetization of collector $\mathbf{M}^{\mathbf{c o l}}$ and emitter $\mathbf{M}^{\mathbf{e m}}$ [Fig. 1(c)], transversal $\left(\mathbf{H}^{\mathbf{T}} \mid \hat{\mathbf{y}}\right)$ and longitudinal $\left(\mathbf{H}^{\mathbf{L}} \| \hat{\mathbf{x}}\right)$ magnetic fields were applied along the two easy axes [Figs. 2(d)-2(g)] [34]. In the pump-probe experiments, the mSHG intensity was measured in transversal and longitudinal geometries for positive and negative $M_{y}$ and $M_{x}$, respectively. From these data, we retrieved $E_{e}, E_{m}$ for each pump-probe delay $t$ and $E_{e 0}, E_{m 0}$ in the absence of pump [35]. In the following, we discuss relative variations $\Delta E_{e} / E_{e 0}$ and $\Delta E_{m} / E_{m 0}$ which are shown in Figs. 3, 4.

In equilibrium, $\mathbf{E}_{\mathbf{e}}=\mathbf{E}_{\mathbf{e}}^{\text {int }}$ and $\mathbf{E}_{\mathbf{m}}=\mathbf{E}_{\mathbf{m}}^{\text {int }}$ originate from the interface dipole polarization [37] $P_{i}^{2 \omega}=\chi_{i j k}^{(2)} E_{j}^{\omega} E_{k}^{\omega}+$ $\chi_{i j k, y}^{(2, m)} E_{j}^{\omega} E_{k}^{\omega} M_{y}^{\text {col }}$, where $i, j, k=x, z$. However, after fslaser excitation, the propagating electrons break the

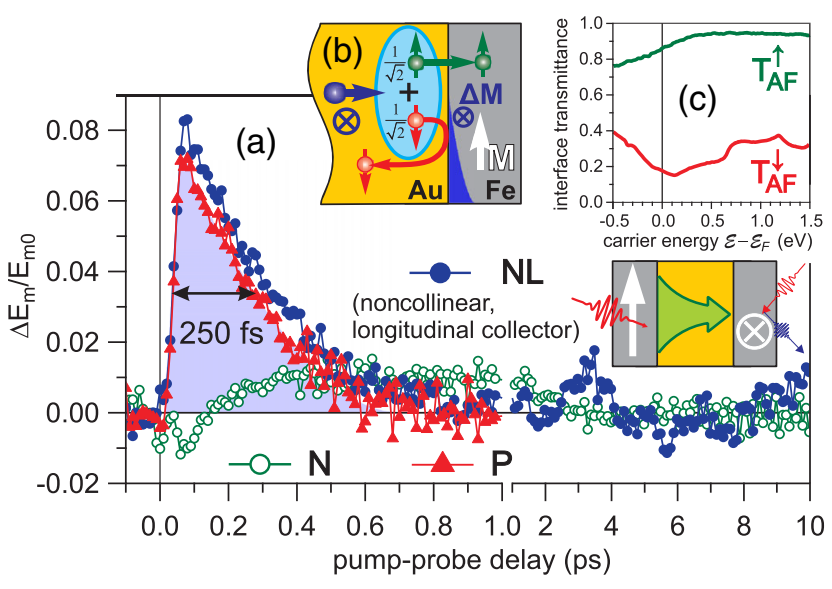

FIG. 4. (a) $\Delta E_{m}^{P}$ and $\Delta E_{m}^{N}$ with the linear background removed represent the SC pulse shape and the contribution of reflected and transmitted hot electrons, respectively. $\Delta E_{m}^{N L}$ differs from $\Delta E_{m}^{P}$ due to the accumulation of the transversal magnetic moment $\Delta M_{T}^{\text {col }}$ in Fe behind the $\mathrm{Au} / \mathrm{Fe}$ interface [cf. (b) and Eq. (2)]. (b) Hot electron behavior at the $\mathrm{Au} / \mathrm{Fe}$ interface in orthogonal ( $N$ and $N L$ ) configurations. (c) Calculated momentum-averaged transmittance of the $\mathrm{Au} / \mathrm{Fe}$ interface for majority $\left(T_{A F}^{\uparrow}\right)$ and minority $\left(T_{A F}^{\downarrow}\right)$ carriers moving from Au to $\mathrm{Fe}$ [36].

inversion symmetry. Thus, bulk dipole current- and SCinduced polarizations $\chi_{i j k z}^{(2, C)} E_{j}^{\omega} E_{k}^{\omega} j_{z}$ and $\chi_{i j k z, y}^{(2, S C)} E_{j}^{\omega} E_{k}^{\omega} j_{z, y}^{S}$ appear [39]. They contribute to $\mathbf{E}_{\mathbf{2} \omega}$ as

$$
\mathbf{E}_{\mathbf{e}}^{\mathbf{C}} \propto j_{z} \propto v_{z}, \quad \mathbf{E}_{\mathbf{m}}^{\mathbf{S C}} \propto j_{z, y}^{S} \propto v_{z} s_{y},
$$

where $j_{z, y}^{S}$ is the SC tensor component with the electron velocity $v_{z}$ and spin polarization $s_{y}$ [40]. Thus, in addition to the modulation of the interface contributions, the total pump-induced variations $\Delta \mathbf{E}_{\mathbf{e}, \mathbf{m}}$ contain bulk terms (1) which provide direct access to the charge and spin currents [41]:

$$
\Delta \mathbf{E}_{\mathbf{e}}=\Delta \mathbf{E}_{\mathbf{e}}^{\text {int }}+\mathbf{E}_{\mathbf{e}}^{\mathbf{C}}, \quad \Delta \mathbf{E}_{\mathbf{m}}=\Delta \mathbf{E}_{\mathbf{m}}^{\text {int }}+\mathbf{E}_{\mathbf{m}}^{\mathbf{S C}} .
$$

This is demonstrated in Fig. 3 where we excite currents from the front $(F)$ or back side of the sample. In the latter case, we consider parallel $(P)$, antiparallel $(A)$, and noncollinear $(N)$ alignment of $\mathbf{M}^{\mathbf{e m}}$ with respect to the transversal $\mathbf{M}^{\mathbf{c o l}}$. First, we discuss the pronounced characteristic features at $t<0.5 \mathrm{ps} \sim \tau_{\mathrm{th}}^{\mathrm{FM}}$. In the $F$ configuration, we observe $\Delta E_{e}^{F}>0$ [Fig. 3(a)] and $\Delta E_{m}^{F}<0$ [Fig. 3(b)]. Then we reverse $\mathbf{v}$ keeping the direction of $\mathbf{s}$ (determined by $\mathbf{M}^{\mathrm{em}}$ ) and find in this $P$ configuration $\Delta E_{e}^{P}<0$ and $\Delta E_{m}^{P}>0$, in agreement with the sign change of $j_{z}$ and $j_{z, y}^{S}$ in Eq. (1). Reversing $j_{z, y}^{S}$ (but not $j_{z}$ ) by keeping $v_{z}$ but changing the sign of $s_{y}$ (A configuration) only slightly affects $\Delta E_{e}$ [Fig. 3(a)] while $\Delta E_{m}$ changes its sign: $\Delta E_{m}^{A}<0$ [Fig. 3(b)]. Lastly, we rotate $\mathbf{s}$ by $90^{\circ}(N$ configuration) to set $j_{z, y}^{S}=0, E_{m}^{S C}=0$ [cf. Eq. (1)] and 
obtain no sizable $\Delta E_{m}^{N}$. Since the major part of $\Delta E_{m}$ changes its sign with reversal of $v_{z}$ or $s_{z}$ (i.e., of $j_{z, y}^{S}$ ), we conclude on the dominant role of $\mathbf{E}_{\mathbf{m}}^{\mathbf{S C}}$ in the mSHG response at short delays. Thus, we unambiguously observe ultrashort SC pulses in Fig. 3(b).

At $t \gg \tau_{\mathrm{th}}^{\mathrm{FM}}$, we expect a reduction of $\mathbf{E}_{\mathbf{m}}^{\text {int }}$ by thermalized electrons heating up the collector: $\Delta \mathbf{E}_{\mathbf{m}}^{\text {int }} \propto \Delta M_{y}^{\text {col }}$. This agrees with the observed $\Delta E_{m}^{P, A, N}(t)$ coinciding at $t>2$ ps [Fig. 3(b)]. Subtracting this trend (solid line) from $\Delta E_{m}^{P}$, we obtain a unipolar trace [Fig. 4(a)] with a sharp onset in contrast to the bipolar behavior of $\Delta E_{m}^{A}$ [Fig. 3(b)]. To understand this difference and its relation to the SC profile [41], we discuss the interaction of spin-polarized electrons with the $\mathrm{Au} / \mathrm{Fe}$ interface [Fig. 4(b)] determined by $T_{A F}$ [Fig. 4(c)]. After considering open $(P)$ and closed $(A)$ states of the spin valve important for GMR devices, we turn to $N$ and $N L$ (noncollinear, longitudinal collector) configurations with $\mathbf{M}^{\mathrm{em}} \perp \mathbf{M}^{\mathbf{c o l}}$ having high potential functionality for spin transfer torque applications [42].

Averaging the calculated interface transmittance over the energy of emitted electrons we obtain $T^{P}=\left\langle T_{A F}^{\uparrow}\right\rangle \approx 0.95$ and $T^{A}=\left\langle T_{A F}^{\downarrow}\right\rangle \approx 0.25$. Therefore, in the $A$ configuration, the reflected SC modifies $\mathbf{E}_{\mathbf{m}}^{\mathbf{S C}}$ [43] leading to bipolar $\Delta E_{m}^{A}(t)$ similar to that measured at the Au surface [14]. In the $P$ configuration, the $\mathrm{SC}$ is absorbed by the collector [44] and $\Delta E_{m}^{P}(t)$ in Fig. 4(a) represents the temporal profile of incoming SC pulse. For ballistic electrons traveling in $\mathrm{Au}$ with the Fermi velocity $1.4 \mathrm{~nm} / \mathrm{fs}$ [45] and random angular distribution the average propagation time $\tau_{b}$ is $70 \mathrm{fs}$. The maximum of $\Delta E_{m}^{P}(t)$ observed at this delay thus suggests ballistic transport [14] with the length $\lambda_{\mathrm{Au}} \gtrsim$ $100 \mathrm{~nm}$ for electrons at $0.3<\mathcal{E}-\mathcal{E}_{F}<1.5 \mathrm{eV}$ [46]. We conclude that $\Delta E_{m}^{P}(t)$ closely reproduces the dynamics of electron emission. The similarity of its decay time of $250 \mathrm{fs}$ [Fig. 4(a)] to the electron thermalization time $\tau_{\text {th }}^{\mathrm{Fe}} \approx 200 \mathrm{fs}$ [47] strongly corroborates the nonthermal SdSE mechanism considered above. Its high efficiency is evident from the negligibly small contribution of thermal SdSE [4], which is within the noise level here.

We now turn to the $N L$ configuration where the longitudinal $\mathbf{M}^{\mathbf{c o l}}$ excludes demagnetization effects in $\mathbf{E}_{\mathbf{m}}^{\text {int }}$ while the transversal $\mathbf{M}^{\mathbf{e m}}$ provides $\mathbf{E}_{\mathbf{m}}^{\mathbf{S C}} \neq 0$. The striking similarity of $\Delta E_{m}^{P}$ and $\Delta E_{m}^{N L}$ at $t<1$ ps [Fig. 4(a)] indicates a negligible contribution of the spin polarization of reflected electrons. Within the single-particle approach, we treat an electron with spin $\mathbf{s} \perp \mathbf{M}^{\text {col }}$ representing its wave function $\left|\Psi_{\perp}\right\rangle=\left(\left|\Psi_{\uparrow}\right\rangle+\left|\Psi_{\downarrow}\right\rangle\right) / \sqrt{2}$ as a superposition of the spin eigenfunctions [48] corresponding to $\mathbf{s} \uparrow \uparrow \mathbf{M}^{\mathbf{c o l}}$ and $\mathbf{s} \downarrow \uparrow \mathbf{M}^{\text {col }}$ which were discussed above for $P$ and $A$ configurations. Considering diagonal transmission operators, we obtain that the transmittance for electrons with $\mathbf{s} \perp \mathbf{M}^{\mathbf{c o l}}$ is the average of transmittance for electrons with $\mathbf{s} \uparrow \uparrow \mathbf{M}^{\text {col }}$ and $\mathbf{s} \downarrow \uparrow \mathbf{M}^{\mathbf{c o l}}$. A limiting case of $T^{P}=1$ and
$T^{A}=0$ is illustrated in Fig. 4(b): only the spin-up (-down) component of the incoming wave is transmitted (reflected). In other words, all electrons with orthogonal spins interacting with FM rotate their spins into parallel and antiparallel states with equal probabilities. Subsequently, electrons with parallel spins are transmitted and antiparallel reflected; i.e., the two spin components are separated in space like in the Stern-Gerlach experiment. This rotated spin polarization does not contribute to $\Delta E_{m}^{N L}$, which explains the similarity of the latter to $\Delta E_{m}^{P}$.

In the general case, transmittances differ from 1 and 0 [Fig. 4(c)] resulting in nonzero residual $\left(\mathbf{s} \perp \mathbf{M}^{\text {col }}\right)$ spin polarization $s_{\perp}$ in both reflected and transmitted currents [48], $\left|s_{\perp}^{R}\right| \leq \sqrt{R^{P} R^{A}}\left|s_{\perp}\right|$ and $\left|s_{\perp}^{T}\right| \leq \sqrt{T^{P} T^{A}}\left|s_{\perp}\right|$, where $R=1-T$. The rotated components $\left(\mathbf{s} \| \mathbf{M}^{\text {col }}\right)$ are given by $s_{\|}^{R}=\left(R^{P}-R^{A}\right)\left|s_{\perp}\right| / 2$ and $s_{\|}^{T}=\left(T^{P}-T^{A}\right)\left|s_{\perp}\right| / 2$. These $s_{\|}$and $s_{\perp}$ are addressed in $N$ and $N L$ configurations, respectively. Using Eqs. (1)-(2), we obtain $\Delta E^{N}(t)=$ $\left(\Delta E^{P}+\Delta E^{A}\right) / 2$, in line with Ref. [48]. Experimentally, this holds for both $\Delta E_{e, m}$ thus indicating the key role of $s_{\|}^{R}$ in the positive $\Delta E_{m}^{N}$ observed at $0.5<t<2 \mathrm{ps}$. The negligible effect of the spin polarization of reflected electrons in $N L$ configuration agrees well with the calculated $R^{P, A}$ for which $\left|s_{\perp}^{R}\right|$ does not exceed $\left|s_{\perp}\right| / 5$.

The angular momentum conservation upon interaction with the $\mathrm{Au} / \mathrm{Fe}$ interface together with the quantum decoherence of the $\left|\Psi_{\uparrow}\right\rangle+\left|\Psi_{\downarrow}\right\rangle$ superposition $\left(s_{\perp}^{T}\right.$ can be up to $s_{\perp} / 2$ ) within the inelastic mean free path $\lambda_{\mathrm{Fe}}^{\downarrow}<$ $1 \mathrm{~nm}$ [25], leads to the emergence of $\Delta \mathbf{M}^{\mathbf{c o l}} \perp \mathbf{M}^{\text {col }}$ in the vicinity of the interface [Fig. 4(b)]. This ultrafast spatially confined spin transfer torque effect inducing $\Delta M_{y}^{\text {col }}$ at the interface is responsible for the small deviation of $\Delta E_{m}^{N L}$ from $\Delta E_{m}^{P}$ observed in Fig. 4(a) at $t<0.5$ ps. Subsequently, several lowest standing spin wave modes at the frequencies up to $0.6 \mathrm{THz}$ are excited in the collector resulting in the nonmonotonic behavior of $\Delta E_{m}^{N L}$ at $t>1$ ps [Fig. 4(a)], see Ref. [42].

Summarizing, using the high sensitivity of nonlinearoptical probe to the transient inversion symmetry breaking, we have demonstrated the generation of ultrashort spin current pulses in $\mathrm{Fe} / \mathrm{Au} / \mathrm{Fe}$ epitaxial multilayers. The measured pulse shape agrees with the proposed nonthermal spin-dependent Seebeck effect and indicates ballistic transport of spin-polarized electrons in Au. The pulse duration ( $250 \mathrm{fs})$ is determined by the electron thermalization time in $\mathrm{Fe}$. We have shown the large difference in transmittance of the $\mathrm{Au} / \mathrm{Fe}$ interface for the spin-polarized electrons with $\mathcal{E}-\mathcal{E}_{F}<1.5 \mathrm{eV}$. This results in a high spin rotation efficiency of $70 \%$ at the interface, where the transmitted (reflected) current loses its orthogonal spin component and becomes polarized parallel (antiparallel) to the $\mathrm{Fe}$ magnetization. These findings facilitate the development of metal-based sources 
of ultrashort spin current pulses and nondissipative reflective or transmittive spin polarizers and rotators for ultrafast spintronics.

The authors thank T. O. Wehling, A. I. Lichtenstein, P. M. Oppeneer, M. Weinelt, and T. Kampfrath for fruitful discussions and M. Wolf for continuous support. Funding by the Deutsche Forschungsgemeinschaft through ME 3570/1 and Sfb 616, the EU 7th framework program through CRONOS and ACMOL (Grants No. 280879 and No. 618082), and Science Foundation Ireland (Grant No. 14/IA/2624 and No. 16/US-C2C/3287) is gratefully acknowledged.

*Corresponding author.

melnikov@fhi-berlin.mpg.de

†Present address: IMMM UMR CNRS 6283, Université du Maine, Avenue Messiaen, 72085 Le Mans, France.

*Present address: Moscow State Technical University of Radioengineering, Electronics and Automation, Prospect Vernadskogo 78, 119454 Moscow, Russian Federation.

${ }^{\S}$ Present address: National Physical Laboratory, Teddington, TW11 0LW, United Kingdom.

[1] A. Fert and I. A. Campbell, Two-Current Conduction in Nickel, Phys. Rev. Lett. 21, 1190 (1968).

[2] K. Uchida, S. Takahashi, K. Harii, J. Ieda, W. Koshibae, K. Ando, S. Maekawa, and E. Saitoh, Observation of the spin Seebeck effect, Nature (London) 455, 778 (2008).

[3] A. Slachter, F. L. Bakker, J.-P. Adam, and B. J. van Wees, Thermally driven spin injection from a ferromagnet into a non-magnetic metal, Nat. Phys. 6, 879 (2010).

[4] G.-M. Choi, C.-H. Moon, B.-C. Min, K.-J. Lee, and D. G. Cahill, Thermal spin-transfer torque driven by the spindependent Seebeck effect in metallic spin-valves, Nat. Phys. 11, 576 (2015).

[5] W. S. Fann, R. Storz, H. W. K. Tom, and J. Bokor, Electron thermalization in gold, Phys. Rev. B 46, 13592 (1992).

[6] U. Bovensiepen, Coherent and incoherent excitations of the Gd(0001) surface on ultrafast timescales, J. Phys. Condens. Matter 19, 083201 (2007).

[7] M. Lisowski, P. A. Loukakos, U. Bovensiepen, J. Stähler, C. Gahl, and M. Wolf, Ultra-fast dynamics of electron thermalization, cooling and transport effects in $\mathrm{Ru}(001)$, Appl. Phys. A 78, 165 (2004).

[8] J. Hohlfeld, S.-S. Wellershoff, J. Güdde, U. Conrad, V. Jähnke, and E. Matthias, Electron and lattice dynamics following optical excitation of metals, Chem. Phys. 251, 237 (2000).

[9] H.-S. Rhie, H. A. Dürr, and W. Eberhardt, Femtosecond Electron and Spin Dynamics in Ni/W(110) Films, Phys. Rev. Lett. 90, 247201 (2003).

[10] M. Battiato, K. Carva, and P. M. Oppeneer, Superdiffusive Spin Transport as a Mechanism of Ultrafast Demagnetization, Phys. Rev. Lett. 105, 027203 (2010).

[11] M. Battiato, K. Carva, and P. M. Oppeneer, Theory of laserinduced ultrafast superdiffusive spin transport in layered heterostructures, Phys. Rev. B 86, 024404 (2012).
[12] C. Stamm et al., Femtosecond modification of electron localization and transfer of angular momentum in nickel, Nat. Mater. 6, 740 (2007).

[13] G. Malinowski, F. Dalla Longa, J. H. H. Rietjens, P. V. Paluskar, R. Huijink, H. J. M. Swagten, and B. Koopmans, Control of speed and efficiency of ultrafast demagnetization by direct transfer of spin angular momentum, Nat. Phys. 4 , 855 (2008).

[14] A. Melnikov, I. Razdolski, T. O. Wehling, E. T. Papaioannou, V. Roddatis, P. Fumagalli, O. Aktsipetrov, A. I. Lichtenstein, and U. Bovensiepen, Ultrafast Transport of Laser-Excited Spin-Polarized Carriers in $\mathrm{Au} / \mathrm{Fe} / \mathrm{MgO}(110)$, Phys. Rev. Lett. 107, 076601 (2011).

[15] D. Rudolf et al., Ultrafast magnetization enhancement in metallic multilayers driven by superdiffusive spin current, Nat. Commun. 3, 1037 (2012).

[16] E. Turgut et al., Controlling the Competition between Optically Induced Ultrafast Spin-Flip Scattering and Spin Transport in Magnetic Multilayers, Phys. Rev. Lett. 110, 197201 (2013).

[17] T. Kampfrath et al., Terahertz spin current pulses controlled by magnetic heterostructures, Nat. Nanotechnol. 8, 256 (2013).

[18] A. Eschenlohr, M. Battiato, P. Maldonado, N. Pontius, T. Kachel, K. Holldack, R. Mitzner, A. Föhlisch, P. M. Oppeneer, and C. Stamm, Ultrafast spin transport as key to femtosecond demagnetization, Nat. Mater. 12, 332 (2013).

[19] A. Kirilyuk, A. V. Kimel, and T. Rasing, Ultrafast optical manipulation of magnetic order, Rev. Mod. Phys. 82, 2731 (2010).

[20] G. E. W. Bauer, E. Saitoh, and B. J. van Wees, Spin caloritronics, Nat. Mater. 11, 391 (2012).

[21] We have calculated the full spin-dependent transmission matrix between all incoming states in $\mathrm{Fe}$ and the outgoing states in $\mathrm{Au}$ for the scattering region consisting of 12 atomic layers of $b c c \mathrm{Fe}(001)$ interfacing an analogous slab of $f c c$ $\mathrm{Au}(001)$ using the nonequilibrium Green's function technique implemented in the Smeagol code [22,23] at the level of LDA.

[22] J. M. Soler, E. Artacho, J. D. Gale, A. García, J. Junquera, P. Ordejón, and D. Sánchez-Portal, The SIESTA method for $a b$ initio order- $N$ materials simulation, J. Phys. Condens. Matter 14, 2745 (2002).

[23] A. R. Rocha, V. M. Garcia-Suarez, S. Bailey, C. Lambert, J. Ferrer, and S. Sanvito, Spin and molecular electronics in atomically generated orbital landscapes, Phys. Rev. B 73, 085414 (2006).

[24] I. Razdolski, A. Alekhin, U. Martens, D. Bürstel, D. Diesing, M. Münzenberg, U. Bovensiepen, and A. Melnikov, Analysis of the time-resolved magneto-optical Kerr effect for ultrafast magnetization dynamics in ferromagnetic thin films, J. Phys. Condens. Matter 29, 174002 (2017).

[25] V. P. Zhukov, E. V. Chulkov, and P. M. Echenique, Lifetimes and inelastic mean free path of low-energy excited electrons in $\mathrm{Fe}, \mathrm{Ni}, \mathrm{Pt}$, and $\mathrm{Au}$ : Ab initio $\mathrm{GW}+\mathrm{T}$ calculations, Phys. Rev. B 73, 125105 (2006).

[26] At the moment we have experimental evidence for the generation of similar SC pulses at the $h c p-\mathrm{Co} / \mathrm{Au}(111)$ interface. 
[27] B. Y. Mueller and B. Rethfeld, Relaxation dynamics in laser-excited metals under nonequilibrium conditions, Phys. Rev. B 87, 035139 (2013).

[28] R.-P. Pan, H. D. Wei, and Y. R. Shen, Optical secondharmonic generation from magnetized surfaces, Phys. Rev. B 39, 1229 (1989).

[29] K.-H. Bennemann, Nonlinear Optics in Metals (Clarendon Press, Oxford, 1998).

[30] B. A. Ruzicka, L. K. Werake, G. Xu, J. B. Khurgin, E. Y. Sherman, J.Z. Wu, and H. Zhao, Second-Harmonic Generation Induced by Electric Currents in GaAs, Phys. Rev. Lett. 108, 077403 (2012).

[31] L. K. Werake and H. Zhao, Observation of second-harmonic generation induced by pure spin currents, Nat. Phys. 6, 875 (2010).

[32] A. Y. Bykov, T. V. Murzina, M. G. Rybin, and E. D. Obraztsova, Second harmonic generation in multilayer graphene induced by direct electric current, Phys. Rev. B 85, 121413(R) (2012).

[33] A. Zvezdin and V. Kotov, Modern Magnetooptics and Magnetooptical Materials (CRC Press, Boca Raton, 1997).

[34] Figures 2(d)-2(g) show the hysteresis loops measured vs $H^{T}$. For $H^{L}=0$, both $\mathbf{M}^{\text {col }}$ and $\mathbf{M}^{\mathrm{em}}$ switch abruptly, although at different $H^{T}$. For $H^{L} \neq 0$, they rotate in two $90^{\circ}$ steps, which results in plateaus [Figs. 2(d),2(e)] corresponding to $\mathbf{M}^{\text {col,em }} \uparrow \uparrow \mathbf{H}^{\mathbf{L}}$. At the same field $H^{T}$, the MOKE ellipticity appears with its sign determined by the direction of $\mathbf{H}^{\mathbf{L}}$ [Figs. 2(f),2(g)]. The step width determined by $H^{L}$ is set such that we can realize all 16 possible combinations of in-plane $\mathbf{M}^{\mathbf{c o l}}$ and $\mathbf{M}^{\mathbf{e m}}$ parallel to the easy axes.

[35] I. Razdolski, D. G. Gheorghe, E. Melander, B. Hjorvarsson, P. Patoka, A. V. Kimel, A. Kirilyuk, E. T. Papaioannou, and T. Rasing, Nonlocal nonlinear magneto-optical response of a magnetoplasmonic crystal, Phys. Rev. B 88, 075436 (2013).

[36] The difference between $T_{F A}^{\uparrow \downarrow}$ and $T_{A F}^{\uparrow \downarrow}$ is due to different numbers of incoming states in $\mathrm{Fe}$ and $\mathrm{Au}$ to which the number of interface states is normalized.

[37] Here we neglect the bulk quadrupole contribution which was shown to be small in $\mathrm{Au}$ [38].
[38] F. X. Wang, F. J. Rodriguez, W. M. Albers, R. Ahorinta, J. E. Sipe, and M. Kauranen, Surface and bulk contributions to the second-order nonlinear optical response of a gold film, Phys. Rev. B 80, 233402 (2009).

[39] These bulk terms have exactly the same symmetry as their interface counterparts and thus cannot be separated by a choice of polarization geometries.

[40] The related signal originates from the $\mathrm{mSHG}$ probing depth $\sim 10 \mathrm{~nm}$ in the $\mathrm{Au}$ layer adjacent to the Fe film.

[41] Possible dependencies of $\chi_{i j k z}^{(2, C)}$ and $\chi_{i j k z, y}^{(2, S C)}$ on the energy of propagating electrons may result in additional timedependent factors in Eqs. (1). Therefore, the extraction of exact shape of $\mathbf{j}$ and $\mathbf{j}$ 采 pulses is beyond the scope of this Letter. Here we focus on the most pronounced features of the response allowing to conclude on the typical pulse shape and duration.

[42] I. Razdolski, A. Alekhin, N. Ilin, J. P. Meyburg, V. Roddatis, D. Diesing, U. Bovensiepen, and A. Melnikov, Nanoscale interface confinement of ultrafast spin transfer torque driving non-uniform spin dynamics, Nat. Commun. 8, 15007 (2017).

[43] Because of the velocity sign reversal, contributions of incoming and reflected SC have opposite signs, cf. Eq. (1).

[44] The current- and SC-induced contributions from the collector are neglected due to much smaller nonlinearity (almost two orders of magnitude smaller mSHG intensity) and inelastic mean free path in $\mathrm{Fe}$ [25], as compared to Au.

[45] S. D. Brorson, J. G. Fujimoto, and E. P. Ippen, Femtosecond Electronic Heat-Transport Dynamics in Thin Gold Films, Phys. Rev. Lett. 59, 1962 (1987).

[46] This ballistic spin transport is solely defined by the interface transmittance and inelastic mean free path in $\mathrm{Au}$, which can be rather long for single crystalline samples. In our earlier work [14], we have demonstrated the ballistic electron transport across $100 \mathrm{~nm}$ thick $\mathrm{Au}$ films.

[47] A. Alekhin, Ph. D. thesis, Freie Universität Berlin, 2016.

[48] M. D. Stiles and A. Zangwill, Anatomy of spin-transfer torque, Phys. Rev. B 66, 014407 (2002). 